\title{
Constant Envelope OFDM Transmission Over Impulsive Noise Power-Line Communication Channels
}

\author{
K. M. Rabie*, E. Alsusa*, A. D. Familua ${ }^{\ddagger}$, and L. Cheng ${ }^{\ddagger}$ \\ ${ }^{*}$ School of Electrical \& Electronic Engineering, The University of Manchester, Manchester, UK, M13 9PL \\ Email: \{khaled.rabie, e.alsusa\}@manchester.ac.uk \\ ${ }^{\ddagger}$ School of Electrical \& Information Engineering, University of Witwatersrand, Private Bag 3, Wits 2050, \\ Johannesburg, South Africa \\ Email: ayokunle.familua@students.wits.ac.za; ling.cheng@wits.ac.za
}

\begin{abstract}
Signal blanking is a simple and efficient method to reduce the effect of impulsive noise over power-line channels. The efficiency of this method, however, is found to be not only impacted by the threshold selection but also by the average peak-to-average ratio (PAPR) value of the orthogonal frequency division multiplexing (OFDM) signals. As such, the blanking capability can be further enhanced by reducing the PAPR value. With this in mind, in this paper we evaluate the performance of constant envelope OFDM (CE-OFDM) which has inherently the lowest achievable PAPR of $0 \mathrm{~dB}$; therefore, the proposed system is expected to provide the lower bound performance of the blanking-based method. In order to characterize system performance, we consider the probability of blanking error and signal-to-noise ratio (SNR) at the output of the blanking device. The results reveal that the proposed system can achieve significant improvements over the conventional OFDM blanking-based scheme in terms of minimized probability of blanking error. It will also be shown that output SNR gains of up to 6 dB can be attained over the conventional OFDM blanking-based systems.
\end{abstract}

Index Terms-Blanking, constant envelope OFDM (CE-OFDM), peak-to-average power ratio (PAPR), power-line communications (PLC), signal-to-noise ratio (SNR).

\section{INTRODUCTION}

It is commonly known that impulsive noise (IN) is the main factor responsible for degrading communication signals over power-line communication (PLC) channels [1], [2]. Mutlicarrier modulation (MCM) systems, such as orthogonal frequency division modulation (OFDM), are found to be more robust to this noise compared to single-carrier systems due to the property of spreading the noise energy over the available sub-carriers. Such a property, however, could turn into a disadvantage if the noise energy is very high where all sub-carriers become contaminated with high levels of interference. In such noise environments, other techniques, with varying degrees of effectiveness and complexity, should be utilized; the simplest and most effective of which remains the blanking scheme. In this scheme, the receiver is preceded with a blanking device to zero the incoming signal when it exceeds a certain threshold value. Blanking the unaffected useful signals will cause blanking errors and hence performance will degrade. In fact, one of the major causes of blanking errors is the high signal peaks associated with conventional OFDM systems, also known as the peak-to-average power ratio (PAPR) problem.

To reduce the PAPR of OFDM systems, many techniques have been introduced in the literature which are, generally, classified into distortionless techniques such as coding [3], tone reservation [4] and selective mapping (SLM) [5]; and non-distortionless techniques such as signal clipping and peak cancellation [6], [7]. In general, although distortionless techniques are relatively more complex than the non-distortionless ones, they are still more attractive because the distortion caused by non-distortionless schemes can outweigh the benefit of the reduced PAPR. The other alternative technique is based on transforming the OFDM signal prior to transmission and applying the inverse transformation at the receiver prior to demodulation. For instance, a companding transform is studied in [8], [9] where companded signals have an increased average power and, consequently, attains lower PAPR compared to conventional OFDM but, relatively, it is still large compared to single-carrier modulation. On the other hand, the authors in [10]-[12] proposed a phase modulator transform where it is shown that such a system can offer the lowest possible PAPR value of $0 \mathrm{~dB}$. This system is referred to as constant envelope OFDM (CE-OFDM). Unlike conventional OFDM which amplitude modulates the carrier, CE-OFDM uses the OFDM signal to phase modulate the carrier and the inverse transform is performed at the receiver prior to the OFDM demodulator. Phase modulation transforms the amplitude variations into a constant amplitude signal by phase modulating the carrier which results in transforming the high PAPR OFDM signal to a $0 \mathrm{~dB}$ PAPR constant envelope waveform. It should be highlighted that transmitting OFDM by ways of angle modulation to reduce PAPR has been in the literature for a while but it was only studied in the context of power amplifier nonlinearity. In contrast to these studies, in this paper we utilize this property to enhance the capability of the impulsive noise mitigation process in PLC systems.

Therefore, the contribution of this paper is threefold. Firstly, the PAPR performance of the CE-OFDM and its impact on the probability of blanking error are studied. The second contribution resides in evaluating the signal-to-noise ratio (SNR) at the output of the blanking device. Then the problem of blanking threshold optimization is addressed and the corresponding maximum achievable output SNR is presented. For the sake of comparison and completeness, throughout our investigations results for the conventional OFDM blanking-based scheme are included. Results show that CE-OFDM with a blanking device can significantly outperform the OFDM-based system in terms of the probability of blanking error. Furthermore, it is shown that the proposed system 
is able to achieve a gain of up to $6 \mathrm{~dB}$ in the output SNR relative to the conventional OFDM scheme.

The rest of the paper is organized as follows. Section II highlights the key differences between the OFDM and CE-OFDM systems. In Section III, the system model is presented. Section IV evaluates the complementary cumulative distribution function (CCDF) and probability of blanking error performances for both the CE-OFDM and OFDM systems. In Section V the output SNR is studied as a function of the blanking threshold whereas the problem of blanking threshold optimization is addressed in Section VI. Finally, conclusions are drawn in Section VII.

\section{CE-OFDM VERSUS OFDM SIGNALING}

In this section we present the main differences between the CE-OFDM and OFDM systems. To start with, a typical OFDM waveform is represented by

$$
x(t)=\sum_{k=0}^{N-1} X_{k} e^{j 2 \pi k t / T_{B}}, \quad 0 \leq t \leq T_{B}
$$

where $X_{k}$ is the complex constellations of the data symbols, $T_{B}$ is the active symbol interval, $N$ is the number of sub-carriers. Therefore, the real and imaginary parts of the OFDM signal can be expressed as

$$
\begin{array}{r}
\mathfrak{R}\{x(t)\}=\sum_{k=0}^{N-1} \mathfrak{R}\left\{X_{k}\right\} \cos \left(2 \pi k t / T_{B}\right) \\
-\mathfrak{I}\left\{X_{k}\right\} \sin \left(2 \pi k t / T_{B}\right)
\end{array}
$$

and

$$
\begin{array}{r}
\mathfrak{I}\{x(t)\}=\sum_{k=0}^{N-1} \mathfrak{R}\left\{X_{k}\right\} \sin \left(2 \pi k t / T_{B}\right) \\
+\mathfrak{I}\left\{X_{k}\right\} \cos \left(2 \pi k t / T_{B}\right),
\end{array}
$$

respectively, and the instantaneous signal power is given by

$$
|x(t)|^{2}=\mathfrak{R}^{2}\{x(t)\}+\mathfrak{I}^{2}\{x(t)\}
$$

This clearly indicates that the signal power fluctuates over time. On the other hand, the general constant envelope (CE) signal has the following form

$$
s(t)=A e^{j \phi(t)}
$$

where $A$ is the signal amplitude and $\phi(t)$ is its phase. CE-OFDM combines the advantages of both OFDM and CE modulation in which the high PAPR OFDM signal is converted into a constant envelope waveform. CE-OFDM has the form of (5) where the phase signal is a real-valued OFDM waveform which can be generated in various ways. For instance, in [13] the complex OFDM signal is divided into its real and imaginary components prior to the phase modulator whereas in [14] discrete cosine transform (DCT) is used. For this paper, to generate the realvalued OFDM signal we use the Hermitian symmetry to map the symbols prior to the OFDM modulator as will be illustrated in the next section. Since the OFDM signal is used to phase modulate the carrier, unlike the conventional OFDM which amplitude

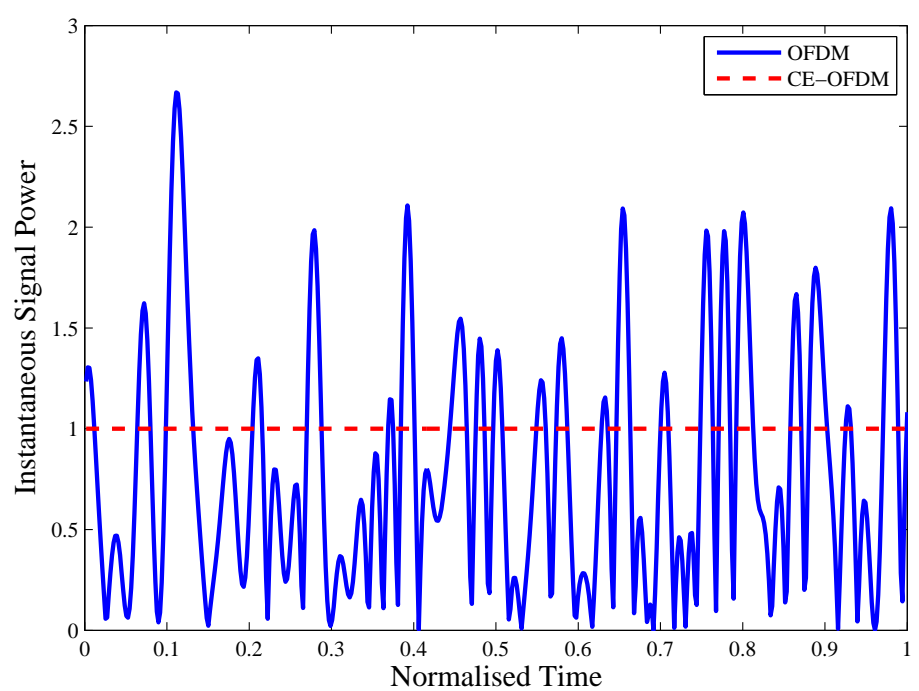

Figure 1: Instantaneous power of CE-OFDM and OFDM signals.

modulates the carrier, the phase signal of CE-OFDM can be expressed as

$$
\begin{aligned}
\phi(t)=\mathfrak{R}\{x(t)\}=\sum_{k=0}^{N-1} \mathfrak{R}\left\{X_{k}\right\} \cos \left(2 \pi k t / T_{B}\right) \\
\quad-\Im\left\{X_{k}\right\} \sin \left(2 \pi k t / T_{B}\right)
\end{aligned}
$$

The CE-OFDM signal power is simply $\left|s_{c e-o f d m}(t)\right|^{2}=A^{2}$, and hence its PAPR is $0 \mathrm{~dB}$. For better clarity, illustrative examples of the instantaneous power of the OFDM and the mapped CE-OFDM signals are presented in Fig. 1 from which it is clear that the peak and the average values of the CEOFDM signal are the same hence its PAPR is $0 \mathrm{~dB}$. It should be mentioned here that the spectral efficiency of CE-OFDM is not as good as conventional OFDM since real-valued signals are required at the input of the phase modulator [11]. Similar to conventional OFDM, CE-OFDM uses cyclic prefix to simplify equalization over multipath fading channels.

\section{SYSTEM MODEL}

The CE-OFDM system model under consideration is shown in Fig. 2 where the unshaded blocks basically represent the conventional OFDM system. In both systems, the information bits are first mapped into 16-QAM symbols, $\mathbf{X}=$ $\left[X_{0}, X_{1}, \ldots, X_{N / 2-1}\right]$, which are then serial-to-parallel (S-toP) converted. For the OFDM system, these symbols are passed directly through an inverse discrete Fourier transform (IDFT) to produce complex valued sequence, $\left(x_{n}^{c v}\right)$, given by

$$
x_{n}^{c v}=\sum_{k=0}^{N-1} X_{k} e^{j 2 \pi k n / N}, \quad n=0,1, \ldots, N-1
$$

However, for the CE-OFDM signal a real-valued time domain OFDM signal is obtained by enforcing Hermitian symmetry in the symbols prior to the IDFT such that

$$
\mathbf{X}=\left[0, X_{1}, \ldots, X_{N / 2-1}, X_{N / 2-1}^{*}, \ldots, X_{1}^{*}, X_{0}^{*}\right]
$$




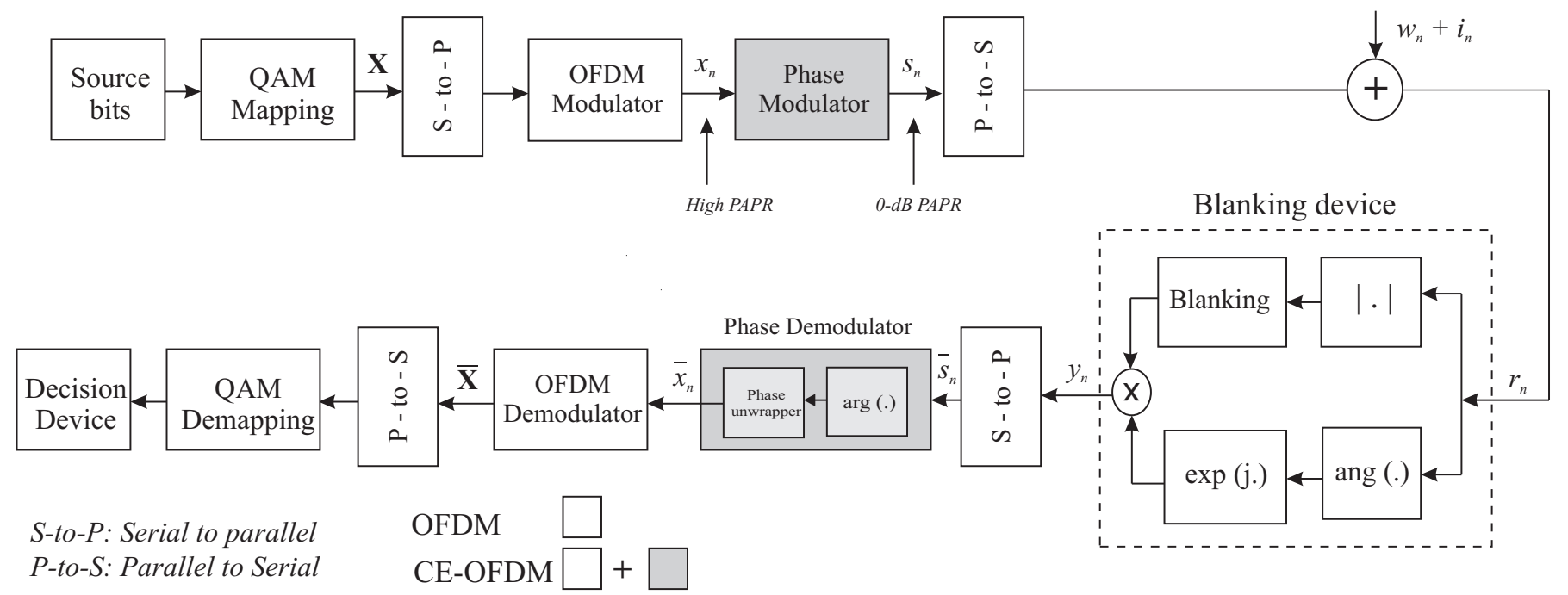

Figure 2: CE-OFDM system model with blanking at the receiver.

The zero at index $k=0$ is used to maintain conjugate symmetry. This sequence is then passed through the $N$-point IDFT to yield a real-valued sequence, $\left(x_{n}^{r v}\right)$, which can be expressed as

$$
x_{n}^{r v}=2 \mathfrak{R}\left\{\sum_{k=1}^{\tilde{N}-1} X_{k} e^{j 2 \pi k n / \tilde{N}}\right\}, n=0,1, \ldots, \tilde{N}-1
$$

where $\tilde{N}=2 N$. The PAPR of the transmitted signal is

$$
\text { PAPR }=\frac{\max \left(\left|x_{n}\right|^{2}\right)}{\mathbb{E}\left[\left|x_{n}\right|^{2}\right]}
$$

After that the real-valued OFDM signal is fed to a phase modulator to produce $s(t)=A e^{j \phi(t)}$, where $A$ is the signal amplitude. In this paper we adopt the two component mixtureGaussian noise model to characterize the total noise in which IN is a result of two random sequences, a real Bernoulli and complex Gaussian process [2], [15]

$$
n_{n}=w_{n}+i_{n} \quad n=0,1,2, \ldots, \tilde{N}-1
$$

where

$$
i_{n}=b_{n} g_{n}, \quad n=0,1,2, \ldots, \tilde{N}-1
$$

while $n_{n}$ is the total noise component, $w_{n}$ is the additive white Gaussian noise (AWGN), $i_{n}$ is the IN, $g_{n}$ is complex white Gaussian noise with mean zero and $b_{n}$ is the Bernoulli process with probability mass function

$$
P\left(b_{n}\right)=\left\{\begin{array}{ll}
p, & b_{n}=1 \\
1-p, & b_{n}=0
\end{array} \quad n=0,1, \ldots, \tilde{N}-1\right.
$$

where $p$ denotes the IN probability of occurrence. The probability density function (PDF) of the total noise can therefore be expressed as

$$
P_{n_{n}}\left(n_{n}\right)=p_{0} \mathcal{G}\left(n_{n}, 0, \sigma_{0}^{2}\right)+p_{1} \mathcal{G}\left(n_{n}, 0, \sigma_{1}^{2}\right)
$$

$\mathcal{G}($.$) is the Gaussian PDF given by \mathcal{G}\left(x, \mu, \sigma_{x}^{2}\right)=$ $\frac{1}{\sqrt{2 \pi \sigma_{x}^{2}}} \exp \left(-\frac{(x-\mu)^{2}}{2 \sigma_{x}^{2}}\right), p_{0}=1-p, p_{1}=p, \sigma_{0}^{2}=\sigma_{w}^{2}$ and $\sigma_{1}^{2}=\sigma_{w}^{2}+\sigma_{i}^{2}$. The variances $\sigma_{w}^{2}$ and $\sigma_{i}^{2}$ denote the AWGN and IN power which define the input SNR and signalto-impulsive noise ratio (SINR) as SNR $=10 \log _{10}\left(1 / \sigma_{w}^{2}\right)$ and SINR $=10 \log _{10}\left(1 / \sigma_{i}^{2}\right)$, respectively. The received signal is then given as

$$
r_{n}=\left\{\begin{array}{ll}
s_{n}+w_{n}, & \mathcal{H}_{0} \\
s_{n}+w_{n}+i_{n}, & \mathcal{H}_{1}
\end{array} \quad n=0,1, \ldots, \tilde{N}-1\right.
$$

where the null hypothesis $\mathcal{H}_{0}$ implies the absence of IN, $P\left(\mathcal{H}_{0}\right)=1-p$, whereas the alternative hypothesis $\mathcal{H}_{1}$ implies the presence of IN, $P\left(\mathcal{H}_{1}\right)=p$. At the receiver's front-end blanking is applied as follows

$$
y_{n}=\left\{\begin{array}{ll}
r_{n}, & \left|r_{n}\right| \leq T \\
0, & \left|r_{n}\right|>T
\end{array} \quad n=0,1, \ldots, \tilde{N}-1\right.
$$

where $T$ is the blanking threshold, $r_{k}$ and $y_{k}$ are the input and the output of the blanking device, respectively. It should be noted that the blanking device only processes the amplitude of the received signal while preserving its phase. Next, $y_{n}$ is fed to the S-to-P convertor and then passed through the phase demodulator where $\arg ($.) calculates the phase of the received samples while the phase unwrapper is used to reduce the effect of phase ambiguities by adding multiples of $2 \pi$ radians where appropriate to eliminate jumps greater than $\pi$ radians.

\section{CCDF And Probability of Blanking ERror}

The PAPR value is a random quantity depending on the input data symbols $X_{n}$ and therefore, its distribution is usually viewed statistically in terms of CCDF [16]. The CCDF of PAPR is defined as the probability that the PAPR of a data block exceeds a given threshold $\left(\mathrm{PAPR}_{o}\right)$ and is expressed mathematically as $\mathrm{CCDF}=\operatorname{Pr}\left\{\mathrm{PAPR}>\mathrm{PAPR}_{o}\right\}$. For accurate estimates of the 


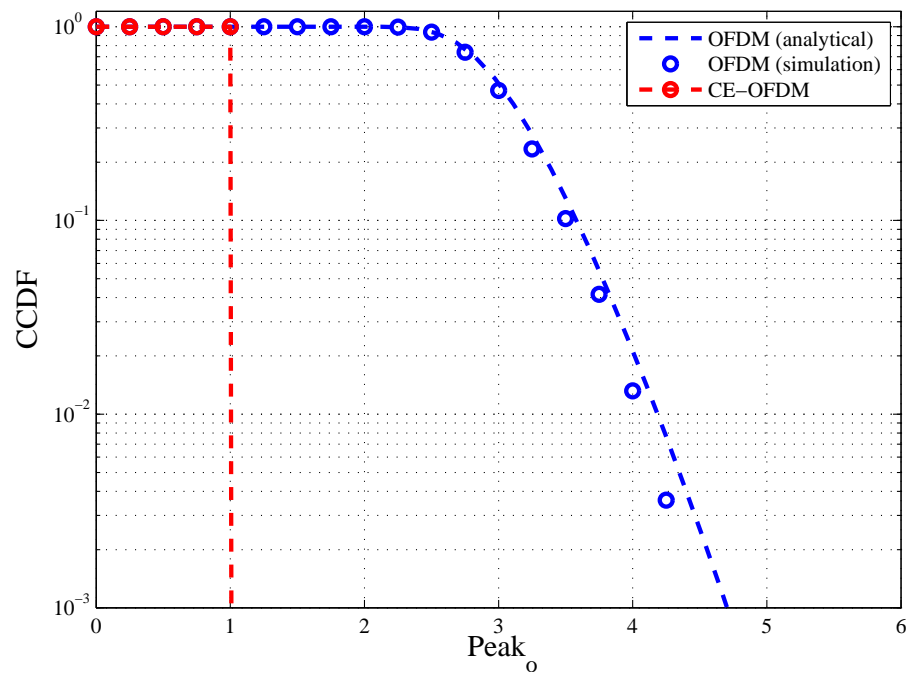

Figure 3: CCDF plot for the CE-OFDM and conventional OFDM systems $(N=64)$.

PAPR, oversampling of 4 is implemented in our investigations. Fig. 3 compares results of the CCDF for the CE-OFDM and conventional OFDM systems with $N=64$. The analytical results of the latter system are found as

$$
\mathrm{CCDF}_{\text {ofdm }}=1-\left(1-\exp \left(-\mathrm{Peak}_{\mathrm{o}}\right)\right)^{N}
$$

From this figure it is evident that the analytical results of the conventional OFDM scheme correlate well with the simulated ones. It is also clearly visible that the CE-OFDM system can drastically reduce the signal peak. For instance, at $\mathrm{CCDF}=10^{-3}$ the proposed system is able to reduce the peak value by 3.5 units compared to OFDM. This PAPR enhancement implies minimizing the probability of blanking error $\left(P_{b}\right) . P_{b}$ is defined as the probability that the received signal, $A_{r}=\left|r_{n}\right|$, exceeds the blanking threshold when it is unaffected by IN and is given mathematically by the following joint probability

$$
P_{b}=P\left(B, \mathcal{H}_{0}\right)=\operatorname{Pr}\left(A_{r}>T \mid \mathcal{H}_{0}\right) P\left(\mathcal{H}_{0}\right)
$$

where $B$ is the event of blanking. It was shown in [17] that the major cause responsible for worsening this probability is the high PAPR of the transmitted signal which is an inherent problem associated with the OFDM waveform. On the contrary, CE-OFDM, as shown above, has the lowest possible PAPR value of $0 \mathrm{~dB}$ allowing to achieve the minimum probability of blanking error performance. To illustrate this, we plot in Fig. 4 some results for $P_{b}$ performance versus $T$ for both the CE-OFDM and conventional OFDM systems. It should be pointed out that the analytical results for the latter scheme are found using [17]

$$
P_{b}=\exp \left(-\frac{T^{2}}{2\left(\sigma_{x}^{2}+\sigma_{w}^{2}\right)}\right)(1-p)
$$

The first observation one can see from these results is that the analytical and simulated results of the conventional OFDM scheme are in a good agreement. It should be stated here that our simulations from this point onward, unless it is clearly stated otherwise, are based on CE-OFDM/OFDM with $N=64$ sub-carriers, oversampling rate of 4 , input $\mathrm{SNR}=25 \mathrm{~dB}$ and

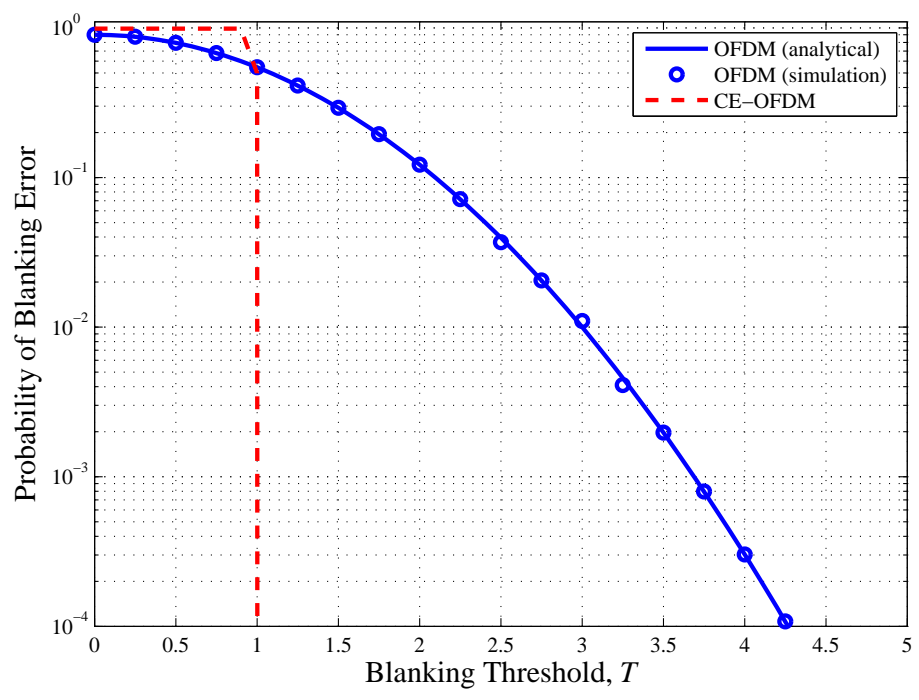

Figure 4: Probability of blanking error versus blanking threshold for the CE-OFDM and OFDM systems.

SINR $=-15 \mathrm{~dB}$. It can clearly be seen from Fig. 4 that the CEOFDM scheme is very robust against blanking errors providing zero probability of blanking error at around 1 whereas in the case of OFDM $P_{b}=0.3$ at the same blanking threshold value and it improves as $T$ increases. It is also observed that when $T<1$, the proposed system has $P_{b}=1$. This is due to the fact that in this region all the signal samples have amplitudes of 1 , as shown in Fig. 1, and will consequently all be blanked. Furthermore, it is worth mentioning that in this region the conventional OFDM system has slightly lower probability of blanking error in comparison to CE-OFDM.

\section{Output SNR as a Function of Blanking Threshold}

This section investigates the impact of blanking on the SNR at the output of the blanking device. Fig. 5 illustrates the output SNR versus $T$ for the CE-OFDM and conventional OFDM systems for various IN probabilities when input $\mathrm{SNR}=25 \mathrm{~dB}$ and SINR $=-15 \mathrm{~dB}$. It is clear that the analytical results of the conventional OFDM system, which are obtained using $[18$, (2) and (26)], and the simulated ones are matching. The simulated output SNR is simply calculated as follows $\mathrm{SNR}_{\text {out }}=$ $\mathbb{E}\left[\left|x_{n}\right|^{2}\right] / \mathbb{E}\left[\left|y_{n}-x_{n}\right|^{2}\right]$. A common observation one can see for both systems is that the output SNR performance degrades as $p$ becomes higher. However, irrespective of the IN probability, CE-OFDM with blanking can generally outperform the OFDMbased scheme when the blanking threshold value is moderate providing a SNR gain of up to $4 \mathrm{~dB}$ and $3 \mathrm{~dB}$ when $p=0.01$ and 0.05 , respectively; whereas when $T$ is very large both systems perform similarly due to the fact that IN will impact both systems in a similar manner in the absence of the blanking device. On the other hand, when $T$ is very small both systems suffer greatly from severe performance degradation since most of the useful signal energy is lost. Furthermore, it is evident that for each system, and for each IN probability, there exists an optimal blanking threshold that maximizes the output SNR. Interestingly enough, however, and unlike the OFDM system in which the maximum achievable output SNR is reached gradually, in the CE-OFDM system the output SNR hits its peak sharply at around $T=1$. This phenomena is of great importance as discussed below. 


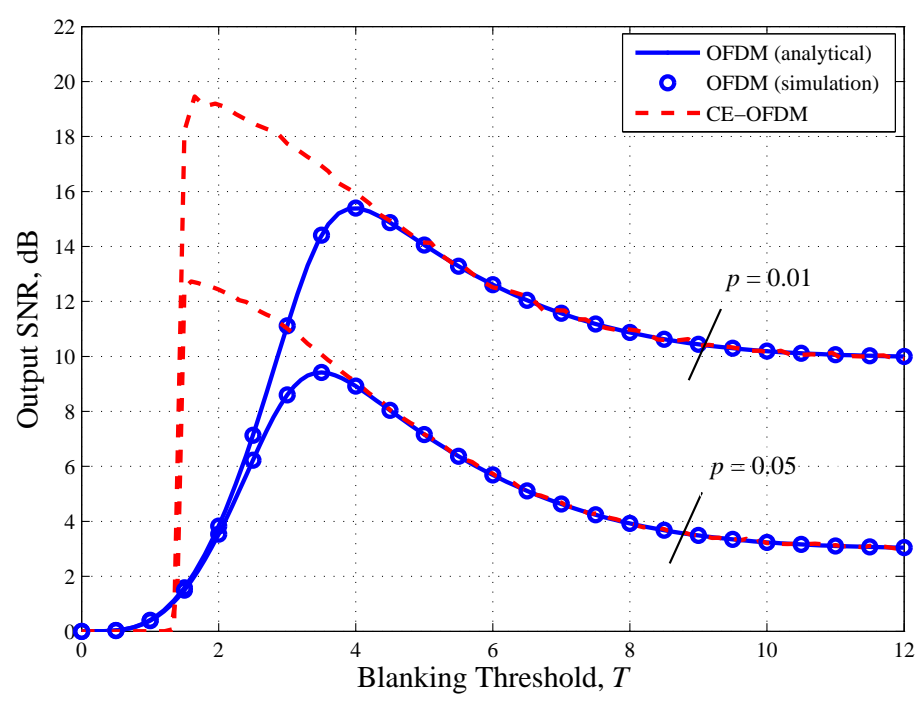

Figure 5: Output SNR performance as a function of the blanking threshold for the CE-OFDM and OFDM systems when $p=0.01$ and 0.05 .

\section{PERFormanCE OPtimization}

In this section extensive simulations are conducted to find the optimal blanking threshold of the proposed scheme as well as the corresponding maximum achievable output SNR in various noise scenarios. To start with, we plot the optimal threshold versus SINR in Fig. 6 for both the CE-OFDM and conventional OFDM systems with $p=0.01$ and 0.1. Comparing the CE-OFDM and OFDM results, it can be seen that the optimal threshold of the former scheme is always lower compared to that of the latter. In addition, unlike conventional OFDM in which the optimal threshold decreases as $p$ becomes higher, in the CE-OFDM approach it remains constant regardless of the value of $p$. Another interesting feature for the proposed scheme is the fact that the optimal threshold is independent of SINR. These advantageous properties of the proposed system imply that optimal performance can always be achieved with no need for prior knowledge about the noise characteristics. This will, consequently, eliminate the requirement for noise estimations and hence estimation errors can be avoided and receiver complexity can also be reduced considerably.

The maximum achievable output SNR for the optimized systems is plotted in Fig. 7 versus SINR for various pulse probabilities. In order to provide comparative results, the output SNR of the conventional OFDM approach is also included on this plot. As a first remark on these results, it is evident that for both modulation schemes the output SNR is reduced as $p$ increases. It is also obvious that the proposed technique always outperforms the conventional OFDM system throughout the SINR spectrum while offering highest gains in the intermediate SINR region $(-3 \mathrm{~dB}>\mathrm{SINR}>-10 \mathrm{~dB})$ of up to $6 \mathrm{~dB}$ and $5 \mathrm{~dB}$ when $p=0.01$ and 0.1 , respectively. However, in the low SINR region (SINR $<-30 \mathrm{~dB}$ ), both systems behave similarly which is intuitively justified by the fact that when noise pulses are extremely high both schemes are able to perfectly detect and cancel the noise pulses. To summarize, CE-OFDM with blanking has the following advantageous properties: a) it has a better performance than conventional OFDM and does not require any noise estimations to combat IN b) As shown in Fig. 2, CE-OFDM

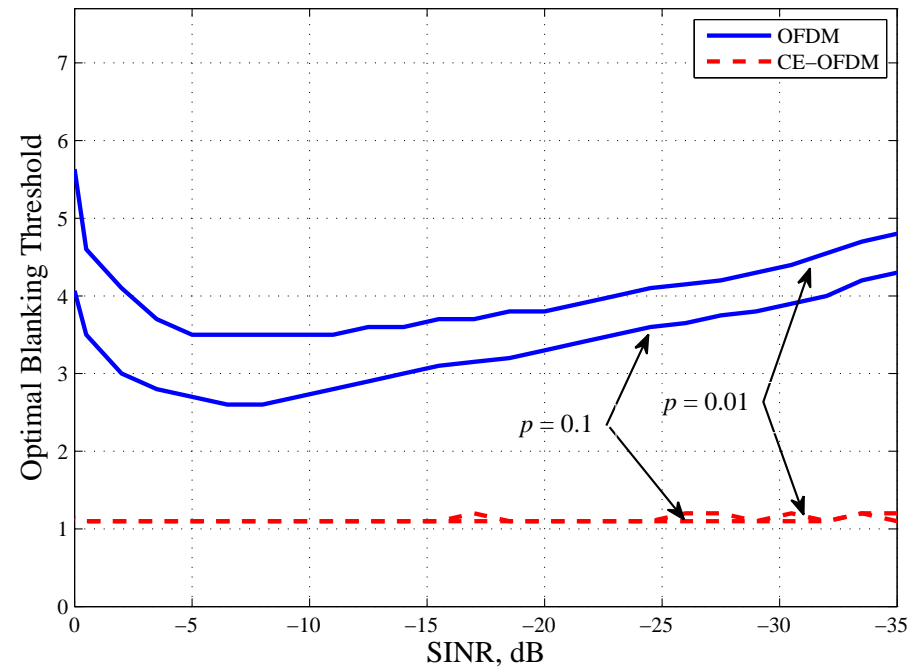

Figure 6: Optimal blanking threshold versus SINR for the CE-OFDM and OFDM systems with various values of $p$.

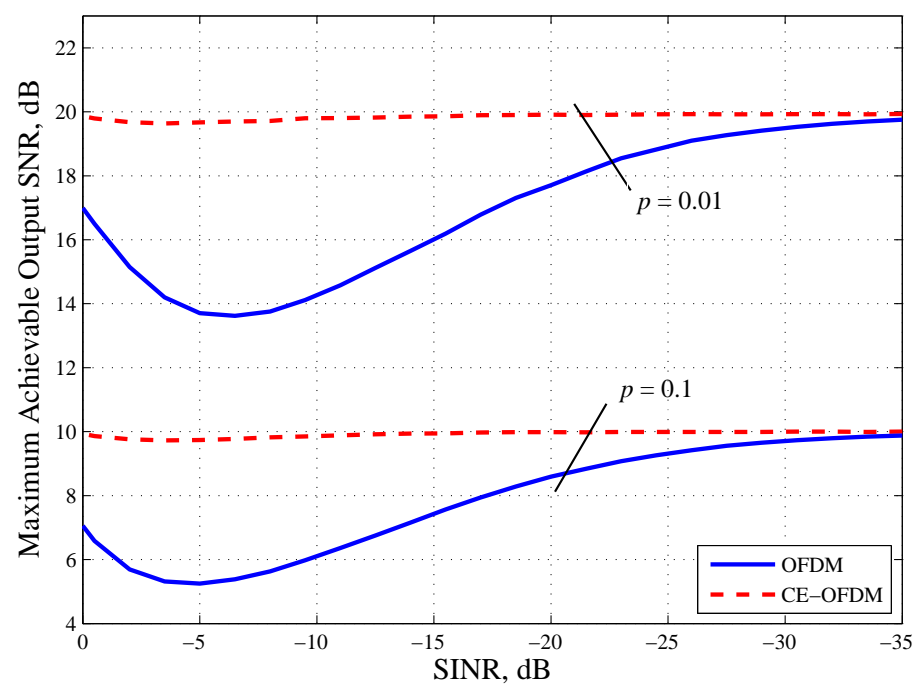

Figure 7: Maximum achievable output SNR as a function of SINR for the CE-OFDM and OFDM systems with different $p$ values.

can be obtained from the conventional OFDM with only minor adjustments.

\section{CONCLUSION}

In this paper we investigated the performance of CE-OFDM with a blanking device over IN power-line channels under various noise conditions. The performance of the proposed system is assessed in terms of the probability of blanking error and output SNR. In addition, the problem of blanking threshold optimization is addressed and the corresponding maximum achievable output SNR is presented. For comparison sake, results for the conventional OFDM based system are demonstrated throughout the paper. It is found that significant improvements can be attained with the proposed technique in the form of considerably minimized probability of blanking error and increased output SNR. Furthermore, it is demonstrated that output SNR gains of up to $6 \mathrm{~dB}$ can be achieved relative to the OFDM system in the intermediate SINR region. 


\section{REFERENCES}

[1] M. Zimmermann and K. Dostert, "Analysis and modeling of impulsive noise in broad-band powerline communications," IEEE Trans. Electromagn. Compat., vol. 44, pp. 249-258, Feb. 2002.

[2] M. Ghosh, "Analysis of the effect of impulse noise on multicarrier and single carrier QAM systems," IEEE Trans. Commun., vol. 44, no. 2, pp. 145-147, Feb. 1996.

[3] J. A. Davis and J. Jedwab, "Peak-to-mean power control in OFDM, golay complementary sequences, and reed-muller codes," IEEE Trans. Inf. Theory, vol. 45, no. 7, pp. 2397-17, Nov. 1999.

[4] J. Tellado, "Peak to average power reduction for multicarrier modulation," Ph.D. dissertation, Stanford Univ. 2000.

[5] R. W. Baaauml, R. F. H. Fisher, and J. B. Huber, "Reducing the peak-toaverage power ratio of multicarrier modulation by selected mapping," Elect. Lett., vol. 32, no. 22, pp. 2056-57, Oct. 1996.

[6] R. O'Neill and L. B. Lopes, "Envelope variations and spectral splatter in clipped multicarrier signals," in Proc. IEEE Pers. Indoor and Mobile Radio Commun. (PIMRC), Toronto, Canada, Sept. 1995, pp. 71-75.

[7] J. Armstrong, "Peak-to-average power reduction for OFDM by repeated clipping and frequency domain filtering," Electron. Lett., vol. 38, no. 5, pp. 246-247, Feb. 2002.

[8] X. Wang, T. Tjhung, and C. Ng, "Reduction of peak-to-average power ratio of OFDM system using a companding technique," IEEE Trans. Broadcast., vol. 45, no. 3, pp. 303-307, Sept. 1999.

[9] X. Huang, J. Lu, J. Zheng, K. Letaief, and J. Gu, "Companding transform for reduction in peak-to-average power ratio of OFDM signals," IEEE Trans. Wireless Commun., vol. 3, no. 6, pp. 2030-2039, Nov. 2004.
[10] R. Pacheco and D. Hatzinakos, "Error rate analysis of phase-modulated OFDM (OFDM-PM) in AWGN channels," in Proc. IEEE Int. Conf. Acoustics, Speech and Signal Process. (ICASSP), vol. 4, May 2006, pp. IV337IV340.

[11] M. Kiviranta, A. Mammela, D. Cabric, D. Sobel, and R. Brodersen, "Constant envelope multicarrier modulation: performance evaluation AWGN and fading channels," in Proc. IEEE Military Commun. (MILCOM), Oct. 2005, pp. 807-813 Vol. 2.

[12] S. Thompson, A. Ahmed, J. Proakis, and J. Zeidler, "Constant envelope OFDM phase modulation: spectral containment, signal space properties and performance," in Proc. IEEE Military Commun. (MILCOM), vol. 2, Oct. 2004, pp. 1129-1135.

[13] C.-D. Chung and S.-M. Cho, "Constant-envelope orthogonal frequency division multiplexing modulation," in Proc. APCC/OECC, vol. 1, Oct. 1999, pp. 629-632.

[14] J. Tan and G. Stuber, "Constant envelope multi-carrier modulation," in Proc. IEEE Military Commun. (MILCOM), vol. 1, Oct. 2002, pp. 607-611.

[15] B. Choi and L. Hanzo, "Adaptive WHT aided QAM for fading channels subjected to impulsive noise," IEEE Commun. Lett., vol. PP, no. 99, pp. $1-4,2013$.

[16] R. van Nee and R. Prasad, OFDM for Wireless Multimedia Communications. Artech House, 2000.

[17] K. Rabie and E. Alsusa, "Preprocessing based impulsive noise reduction for power-line communications," IEEE Trans. Power Del., vol. 29, no. 4, pp. 1648-1658, Aug. 2014.

[18] S. V. Zhidkov, "On the analysis of OFDM receiver with blanking nonlinearity in impulsive noise channels," in Proc. Int. Symp. Intell. Signal Process. Commun. Syst. (ISPACS), Nov. 2004, pp. 492-496. 